\title{
Bericht aus den EAP-Meetings
}

Online, 16.-18. Oktober 2020

Peter Schulthess

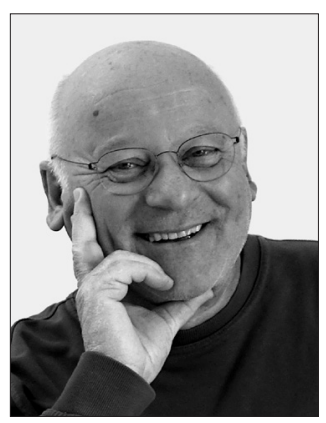

Die Herbstmeetings der EAP fanden diesmal Corona-bedingt als Online-Meetings via Zoom statt. Für die ASP nahm Gabriela Rüttimann an jenem Teil der Meetings teil, der für unseren Verband relevant ist. Ursprünglich hätten die Meetings in Pristina (Kosovo) stattfinden sollen, gefolgt von einer Tagung über die Situation der Flüchtlinge in Europa und mit der Frage, wie PsychotherapeutInnen Support geben können. Dieses Thema ist derzeit brandaktuell, die Tagung musste jedoch aufgrund der Corona-Lage auf nächstes Jahr verschoben werden. Eine Online-Tagung wollten die OrganisatorInnen (das Committee for Refugees) nicht durchführen. Für InteressentInnen: Diese Tagung ist nun für den 24. Oktober 2021 in Pristina vorgesehen.

Das Setting, die Meetings mit bis zu 50 Personen online zu halten, war herausfordernd, ging es doch um tägliche mehrstündige Präsenz vor dem Computer über drei Tage hinweg. Es war aber sicher besser, die Meetings wenigsten online zu halten, um anstehende Geschäfte zu erledigen.

Zum Thema «Forschung» ist zu berichten, dass die EAP auf eine Ausschreibung der EU reagiert und sich mit Forschungspartnern für ein Projekt beworben hat, das zu Händen der europäischen Länder eine situationsspezifische Bewusstheit für mögliche Krankheitsausbrüche mit grösserer Auswirkung auf die Bevölkerung erhöhen und den Regierungen Grundlagen über die soziale Komplexität zum Handeln in solchen Krisen hätte liefern sollen. Das Forschungsprojekt wäre über drei Jahre gelaufen und hätte circa 240.000 Euro gekostet (getragen von der EU). Leider verpasste die EAP-Bewerbung um wenige Punkte den Zuschlag seitens der EU, andere Bewerber machten das Rennen. Für die EAP ist es das erste Mal, dass man sich im Rahmen der EU-Forschungsförderung mit einem Projekt bewarb. Solche Eingaben sind komplex und wir haben für ein nächstes Mal viel gelernt.

Die EAP hat auch den Präsidenten des EU-Parlamentes angeschrieben und dem Wunsch Ausdruck gegeben, dass PsychotherapeutInnen in Anwendung des Common Training Frameworks (CTF) in den EU-Ländern gegenseitig anerkannt würden, entsprechend einer kürzlich erlassenen Direktive des Parlamentes. Die Reaktion war positiv und aufmunternd. Der Präsident erkannte die wichtige Rolle der Psychotherapie im Mental Health-Sektor an.

Beide diese Initiativen wurden von der designierten nächsten EAP-Präsidentin Pat Hunt umgesetzt, letztere in Zusammenarbeit mit der EU-Lobbying Group. Man darf ihrer Präsidentschaft mit Optimismus hinsichtlich weiterer Projekte entgegensehen.

In verschiedenen Ländern laufen derzeit Gesetzgebungsverfahren zur Regelung der Psychotherapie. Zu Händen der spanischen KollegInnen hat die EAP eine Bestätigung verschickt, dass Bioenergetische Therapie von der EAP als vollwertiges Psychotherapieverfahren anerkannt ist. In Rumänien bildet sich eine analoge Situation ab wie seinerzeit in der Schweiz: Die Psychologieverbände kämpfen dafür, dass Psychotherapie als psychologischer Beruf geregelt wird, während die Verbände, die der EAP angehören, eine Regelung wünschen, die Psychotherapie als eigenständigen wissenschaftlichen Beruf regelt. Ähnliches spielt sich auch in Spanien ab. Die EAP hat beide Psychotherapieverbände mit Argumentationshilfen unterstützt. So sieht es eigentlich auch die EU: Die European Federation of Psychologists' Associations (EFPA) wird als zuständige NAO für die Psychologieberufe anerkannt, die EAP als zuständige NAO für Psychotherapie. Wie so oft, will die EFPA diese Abgrenzung nicht einhalten und beansprucht, auch für die Psychotherapie zuständig zu sein. Es bleibt zu hoffen, dass die Revision des Psychotherapiegesetzes in Deutschland, das Direktstudiengänge in Psychotherapie ermöglicht, einen Impact hat, der es erlaubt, Psychotherapie als eigenständigen universitären Beruf zu sehen.

Im National Umbrella Organisations Committee (NUOC) zeigte sich, dass in verschiedenen Ländern Konflikte herrschen, entweder mit einem konkurrierenden Verband oder gar innerhalb des eigenen Verbandes (Polen, Zypern, Tschechien, Irland). Die EAP stellt sich in solchen Konflikten als Schlichtungsstelle zur Verfügung, oft aber mit wenig Erfolg, da die Fronten zu verhärtet sind. Um auch AbsolventInnen der Direktstudiengänge aufnehmen zu können, wurde dem EAPBoard der Antrag eingereicht, die Strassburg Deklaration entsprechend zu ergänzen. Diese Diskussion ist noch nicht abgeschlossen und wird kontrovers geführt. Die einen sehen die Deklaration als unveränderbares historisches Gründungsdokument der EAP, andere finden, das könne verändert werden, so wie jede Verfassung. Und wieder andere meinen, die Deklarati- 
on sei bezüglich Ausbildung so offen abgefasst, dass es gar keine Änderung bräuchte.

Im Februar wurde Regina Hilbert als Assistentin des Generalsekretärs gewählt, insbesondere um das Office der EAP besser zu führen. Das zog jedoch Konflikte nach sich, da ein Pflichtenheft erst nachträglich erstellt wurde. Dieses sah vor, dass Regina Hilbert bloss für die Arbeit zweier Sekretärinnen zuständig war, während die dritte der Registrarin zugeordnet wurde. Diese Splittung der Verantwortung zeigte sich als unbefriedigend und Regina Hilbert kündigte ihre Position nach nur einem halben Jahr.

Im Training Accreditation Committee wurde das einzige Schweizer Institut, das der EAP angehört, das International Institute for Biosynthesis (IIBS) reakkreditiert für weitere sieben Jahre. Das Gesuch wurde auch von der ASP unterstützt.

Das Ethics Committee will die ethischen Richtlinien ergänzen um einen Punkt, der das «copy- righten» von Therapieverfahren ausschliessen will. Der Antrag war noch nicht abstimmungsfähig und wird im März in Wien weiter besprochen. Das International Journal for Psychotherapy musste aufgrund einer Budgetkürzung auf eine Online-Zeitschrift umgestellt werden. Printversionen werden nur noch in kleinen Mengen hergestellt.

Die Finanzlage des Verbandes sieht besser aus als erwartet, bleibt aber dieses Jahr erneut defizitär. Einerseits «half» die COVID-19-Pandemie, Kosten zu sparen, andererseits gingen mehr Mitgliederbeitragszahlungen ein als erwartet. Die elektronische Rechnungsstellung hat dazu beigetragen. Der Verband lebt weiterhin von seinen Reserven und muss, um wieder positive Rechnungsabschlüsse zu erreichen, weitere Einkünfte generieren.

Peter Schulthess ist Vorstandsmitglied der ASP.

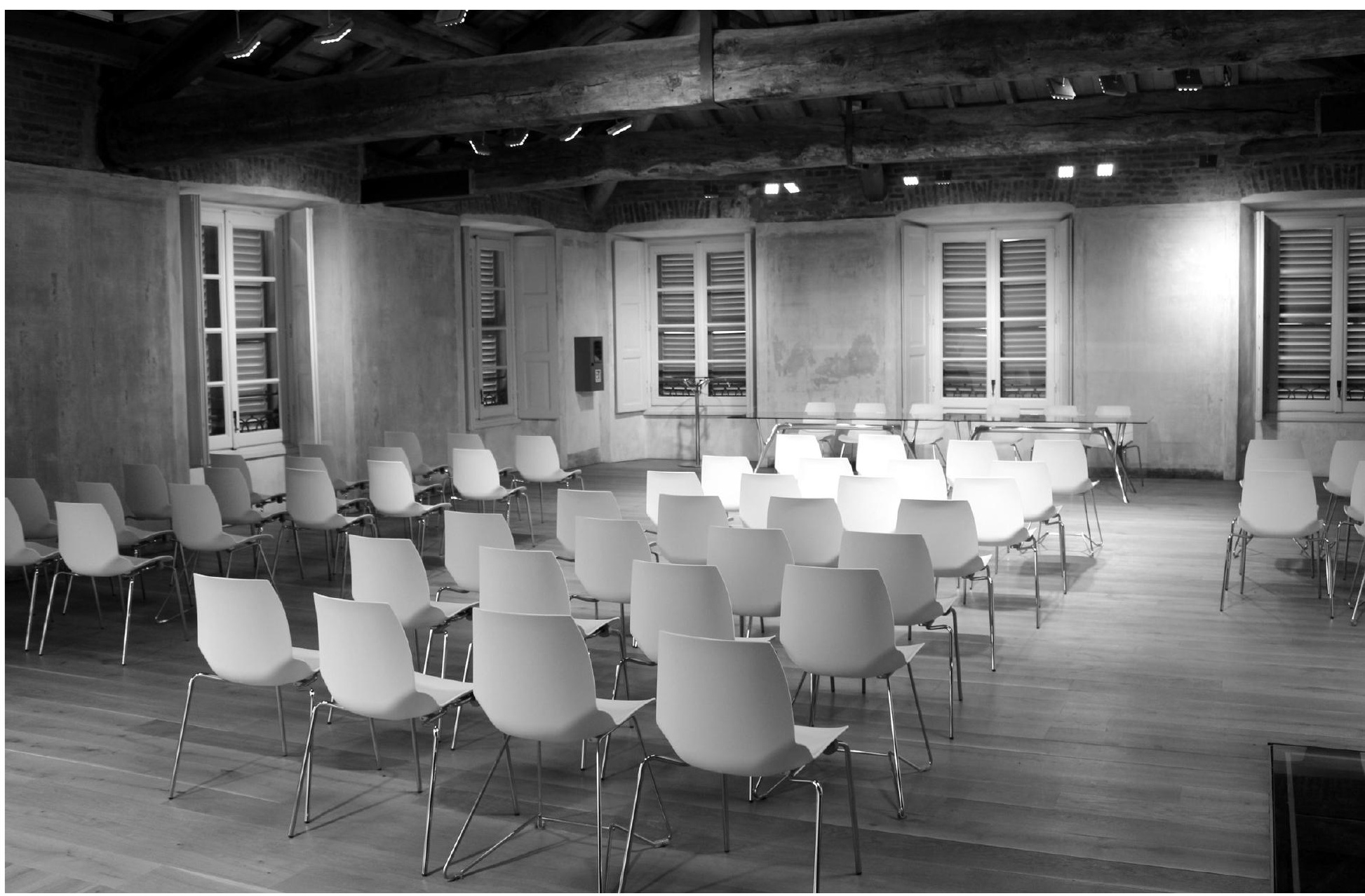

